\title{
Whether idiopathic hirsutism is associated with an increase in metabolic disturbances?
}

Behboudi-Gandevani S, Ramezani Tehrani F,Simbar M, Azizi F.

Reproductive Endocrinology Research Center, Research Institute for Endocrine Sciences, Shahid Beheshti University of Medical Sciences

\section{Introduction}

Idiopathic Hirsutism (IH) is one of the most frequent complaints affecting $13 \%$ of reproductive aged women. The relationship between $\mathrm{IH}$ and metabolic abnormalities are not fully understood. The aim of this study was to evaluate the relationship between $\mathrm{IH}$ and metabolic abnormalities.

\section{Methods}

A total number of 100 Idiopathic hirsute women (range 15-45 years) recruitment from a population based cohort study of Tehran Lipid and Glucose Study. Control age matched women were selected from the same population. Biochemical characteristics of the participants including waist and hip circumferences and ratio, blood pressure, glucose, androgens and lipid profiles and insulin resistance by HOMA-IR were compared. Differences between the two groups in univariate analysis were detected by independent Student's t-test for continuous variables with normal distribution or by the Mann-Whitney U test for those without normal distribution. Analysis of covariance (ANCOVA) was used for comparison of groups with adjustment for the BMI.

\section{Results}

The IH subjects had higher BMI and waist to hip circumferences ratio compared with healthy controls, $25.4(3.2)$ and $0.83(0.04)$ in IH vs. $23.9(4.2)$ and $0.81(0.05)$ in controls $(\mathrm{p}=0.02$ and 0.04$)$, respectively. The mean fasting insulin, HOMA-IR and TG in IH women were statistically higher than in controls but there were not any statistical differences between them and other biochemical, anthropometric, lipid and blood pressure profiles after adjustment for age and BMI.

\section{Conclusions}

Our data suggest that $\mathrm{IH}$ without PCOS did not associate with metabolic disturbances. It seems that no more additional intervention and evaluation are needed in IH subject later in life.

\section{Bibliography}

. SomaniN, Harrison S,Bergfeld WF (2008) The clinical evaluation of hirsutism. Dermatol Ther 21: 376-391.

2. Tehrani FR, Rashidi H, Azizi F (2011) The prevalence of idiopathic hirsutism and polycystic ovary syndrome in the Tehran Lipid and Glucose Study. Reprod Biol polycystic ovary sy $140.1186 / 1477-7827-9-144$

Tehrani FR, Simbar M, Tohidi M, Hosseinpanah F, Azizi F (2011) The prevalence of polycystic ovarysyndrome in a community sample of Iranian population: Iranian PCOS prevalence study. Reprod Biol Endocrinol 9: 39. 